\title{
Modelo de pertinencia y factibilidad académica de la Universidad Autónoma de Tamaulipas
}

\author{
LLÁDO LÁRRAGA, Dora María ${ }^{1}$ \\ LÓPEZ MOCTEZUMA, Cresencio ${ }^{2}$ \\ AZUELA FLORES, Cristabell ${ }^{3}$
}

\section{RESUMEN}

Derivado de las políticas institucionales, nacionales, internacionales, la UAT definió el Modelo de pertinencia y factibilidad académica con la finalidad de fundamentar los procesos de actualización y diseño de nuevas opciones profesionales. El objetivo del presente trabajo es dar a conocer los elementos del modelo así como los resultados preliminares de los estudios realizados en cada uno de los actores que conforman dicho modelo. La metodología empleada en los estudios es de corte cuantitativa, descriptiva, transversal y no experimental, teniendo como sujetos de estudio a estudiantes de nivel medio superior que cursaban su último periodo escolar en mayo de 2019; los egresados del periodo escolar 2019-2; y los empleadores de los egresados de la UAT. Los resultados del estudio de estudiantes evidenció que en su mayoría tienen interés en continuar sus estudios superiores, que un $53,6 \%$ están interesados en las carreras de la UAT, y que las 10 carreras de mayor interés son Enfermería, Ing. Industrial, Medicina, Contador Público, Sistemas Computacionales, Agronomía, Psicología, Negocios Internacionales y Derecho. En el caso de los empleadores y los egresados de la Universidad, se observa coincidencia con respecto a las Competencias, Conocimientos y Habilidades Técnicas que se requieren en el campo laboral.

Palabras clave: pertinencia, factibilidad, estudiantes, egresados, empleadores.

\section{Academic relevance and feasibility model of the Universidad Autónoma de Tamaulipas}

\begin{abstract}
Derived from institutional, national and international policies, the Universidad Autónoma de Taulipas (UAT) defined the Academic Relevance and Feasibility Model in order to provide a basis for the processes of updating and designing new professional options. The aim of this paper is to present the elements of the model, as well as the preliminary results of the studies carried out on each of the participants that make up the model. The methodology used in the studies was quantitative, descriptive, cross-sectional and nonexperimental, having as subjects of study upper middle school students who were in their last school term in May 2019, the graduates of the 2019-2 school term, and the employers of UAT graduates. The study results about the students showed that most of them are interested in continuing their higher education, that $53.6 \%$ are interested in the careers offered by the UAT, and that the 10 careers of higher interest are Nursing, Industrial Engineering, Medicine, Accounting, Computer Systems, Agronomy, Psychology, International Business and Law. As far as the employers and university graduates are concerned, there is a coincidence in terms of the technical skills, knowledge and abilities required in the field of employment.
\end{abstract}

Keywords: relevance, feasibility, students, graduates, employers

'Universidad Autónoma de Tamaulipas. ORCID: 0000-0003-2368-3695. dllado@docentes.uat.edu.mx 


\section{INTRODUCCIÓN}

La UNESCO (Organización de las Naciones Unidas para la Educación, la Ciencia y la Cultura, 2015) establece que si bien el problema de desempleo no es un tema que la educación por sí sola pueda resolver, puede idear planteamientos nuevos en la adquisición de educación y capacidades que capitalicen plenamente el potencial de los egresados de nivel superior.

Las instituciones de educación superior (IES), y en el caso particular de la Universidad Autónoma de Tamaulipas (UAT), deben estar alertas ante las demandas emanadas por la globalización económica, las implicaciones de la formación de los sujetos ante los retos de la sociedad de la información y del conocimiento y ante las formas de operar de los mercados laborales flexibles (Lladó Lárraga, Navarro Leal, \& Sánchez Rodríguez, 2013). Con base en lo anterior, su función sustancial no se concibe sin la posibilidad de identificar el grado en que los programas de estudio permiten a los egresados insertarse exitosamente en el mercado profesional. Igualmente importante para ello, es conocer de primera mano, la opinión de los empleadores y expertos sobre la pertinencia de la oferta educativa y cómo, desde su perspectiva, es posible mejorar su calidad y lograr una mayor concatenación con el medio laboral.

A raíz de resultados de las evaluaciones efectuadas por organismos acreditadores externos, desde hace al menos cinco años, la UAT ha tenido dos observaciones relacionadas con el tema de la pertinencia académica y factibilidad:

a)diseñar y poner en marcha un programa permanente de seguimiento de la trayectoria profesional de los egresados a fin de identificar sus necesidades de actualización tanto académica como profesional y, que a la vez, sirva de base para el rediseño curricular en función de lo que demandan los egresados y los empleadores;

b) fortalecer los canales de comunicación con las empresas empleadoras para conocer cuál es el profesional que requieren y con ello identificar las necesidades del mercado laboral y de la sociedad, con la finalidad de lograr la pertinencia académica en las instituciones educativas. En concordancia con lo anterior, el Plan de Desarrollo Institucional 2018-2021, establece como una de sus políticas prioritarias lograr mayor cobertura, pertinencia y calidad de la oferta educativa. Aunado a ello, una de las estrategias empleadas por la Universidad ha sido la realización de estudios que permitan un acercamiento al conocimiento de las demandas de formación profesional en su contexto inmediato. El diagnóstico realizado en las Facultades y Unidades Académicas, para valorar las experiencias en torno a pertinencia, cobertura y calidad, denotó un desarrollo metodológico aislado y poco sistematizado con relación a los estudios que se venían efectuando en las Facultades de la Universidad, y de ahí la necesidad de establecer institucionalmente el modelo de pertinencia y factibilidad académica.

El objetivo de este escrito es presentar el modelo de pertinencia y factibilidad académica que desarrolló la Universidad a principios de 2019 así como los resultados preliminares de estudios realizados en el contexto de dicho modelo en el transcurso del mismo año.

La primera parte del documento plantea dos conceptos básicos del modelo: pertinencia y factibilidad; se describen los objetivos del modelo y se enmarcan éstos en el contexto de la institución, detallando las políticas educativas en las que se fundamenta el modelo y finalmente se hace una descripción de cada uno de los elementos del mismo.

La segunda parte del trabajo integra los elementos metodológicos y los resultados de investigación preliminar de los estudios realizados con algunos de los actores que integran el modelo de pertinencia que se propone: estudio de expectativas de formación profesional de los estudiantes próximos a egresar del nivel medio superior de Tamaulipas y resultados de los egresados y empleadores. La fase de factibilidad del modelo aún no se desarrolla con las Facultades y Unidades Académicas de la UAT, en virtud de que depende de los resultantes de la etapa de pertinencia, la cual en este momento se encuentra en fase de socialización con las mismas, para la toma de decisiones en torno a cuáles nuevas opciones educativas aperturar.

Finalmente se integra un apartado conclusivo que apunta a la estrategia que actualmente desarrolla la Universidad con miras a lograr mayor pertinencia, calidad y cobertura. 


\section{EL MODELO DE PERTINENCIA Y FACTIBILIDAD ACADÉMICA}

Para la UAT un programa educativo se considera pertinente cuando su fundamentación esté sustentada en los resultados de estudios tales como: expectativas de formación profesional de estudiantes de nivel medio superior y los estudios de opinión de egresados, empleadores y expertos. Asimismo cuando en el proceso de creación o actualización curricular se hayan considerado las demandas de los planes de desarrollo y los resultados de evaluaciones de organismos evaluadores externos. Por otro lado, se considera factible cuando haya efectuado estudios de proyección de demandas de educación superior así como el diagnóstico de las necesidades de infraestructura y recursos humanos, materiales y financieros, que aseguren la viabilidad de la nueva propuesta curricular, ya sea nueva creación o actualización curricular, en la Universidad.

La realización de los estudios de pertinencia y factibilidad se constituyen en una prioridad para el Estado de Tamaulipas y para la Universidad en la medida en que se busca atender, a través de la formación de profesionistas, las demandas de los distintos sectores y fortalecer los polos de desarrollo de Tamaulipas; todo lo anterior, mediante un sustento metodológico fortalecido.

El modelo de pertinencia y factibilidad académica de la Universidad Autónoma de Tamaulipas encuentra su fundamento institucional en el Plan de Desarrollo 2018-2021 (Universidad Autónoma de Tamaulipas, 2018, 14-35) de la actual administración rectoral. Su Misión plantea la importancia de "formar técnicos, artistas y profesionales útiles a la sociedad... con sentido de pertenencia e identidad nacional"; asimismo señala en su Visión "ser una universidad incluyente, equitativa y socialmente responsable, protagonista con el desarrollo socioeconómico y ambiental del estado", estableciendo el compromiso con el Estado de Tamaulipas y con el país, de formar los profesionales que sean demandados para el desarrollo de los sectores potenciales de crecimiento en el estado y para atender de forma pertinente las problemáticas emanadas de su contexto inmediato y mediato. En congruencia con lo anterior, se definieron en su Plan de Desarrollo Institucional (Universidad Autónoma de Tamaulipas, 2018, 14-35) tres ejes estratégicos: Cobertura con equidad, Programas de estudios pertinentes y de calidad y Vinculación.
El modelo que aquí se presenta tiene las siguientes líneas de acción:

a) "Realizar estudios de pertinencia, identificando los nuevos ámbitos de desempeño profesional y las necesidades formativas de los futuros profesionistas".

b) "Realizar estudios prospectivos que sirvan de marco de referencia para la elaboración de estudios de pertinencia y factibilidad".

c) "Actualizar los planes y programas de estudio acorde al modelo educativo y a las necesidades de los sectores empleadores y nuevas tendencias nacionales e internacionales".

El modelo establece dos etapas de trabajo: estudios de pertinencia y estudios de factibilidad. Para realizar los estudios de pertinencia académica en la Universidad Autónoma de Tamaulipas (UAT), se considera la opinión de cuatro tipos de sujetos: estudiantes de media superior, egresados, empleadores y expertos.

a) Estudiantes de media superior: son aquellos jóvenes que se encuentran estudiando el último periodo escolar en el Nivel Medio Superior de cualquier subsistema educativo público o privado. Esta etapa del modelo recolecta las expectativas que los estudiantes plantean con respecto a su futura formación profesional, las carreras e instituciones de su preferencia.

b) Egresados: se refiere a los jóvenes que ya egresaron de la UAT, por lo menos hace tres meses de cualquier programa de Licenciatura. Se busca conocer su percepción sobre la formación profesional así como sobre la situación en el ámbito laboral. Específicamente datos laborales como mecanismos de inserción, puesto, contratación tipo y tamaño de empresa, datos académicos y su nivel de satisfacción con la formación recibida

c) Empleadores: son las instituciones públicas y privadas que cuentan con personal egresado de la UAT. A través de la opinión de empleadores se busca conocer los mecanismos de selección de los profesionistas, sus necesidades formativas, los perfiles deseables, las aptitudes y habilidades requeridas en sus 
potenciales empleos.

d) Expertos: Son profesionistas destacados por su formación académica y/o por su trayectoria profesional, que se reconocen como investigadores del campo de formación disciplinar o bien por las aportaciones que han dado en su desarrollo profesional en los campos de trabajo en que se han desempeñado. Conocen los desafíos actuales y escenarios futuros en torno al desarrollo proyectado en el estado, municipio y la región para identificar las demandas específicas vinculadas al tipo de conocimiento y formación que debieran tener los futuros profesionistas.

Por lo que respecta a la etapa de los estudios de factibilidad, esta requiere considerar:

a) Estudios retrospectivos y prospectivos de la población escolar de nivel medio superior en los últimos 5 años con proyección a 5 años delante. Este estudio tiene la finalidad de analizar la evolución y comportamiento de la población escolar a fin de determinar o asegurar la demanda educativa de los próximos 5 años en la educación superior.

b) Análisis de la capacidad académica de cada programa educativo. Esta fase de trabajo requiere del análisis de los perfiles de la planta académica actual y los requerimientos de profesionalización en los próximos 5 años. Asimismo, establecer las necesidades de incorporación de nuevos perfiles de docentes acorde a las necesidades de los programas educativos en los próximos 5 años, estimando el costo de los mismos.

c) Análisis de equipamiento e infraestructura. Es importante contar con datos actuales del tipo de equipamiento e infraestructura. Con relación al equipo y mobiliario considerar el tiempo de vigencia u obsolescencia en 5 años; de tal modo que en el análisis se deben estimar los costos anuales para la actualización y/o adquisición de los mismos. En cuanto a la infraestructura se debe acompañar de proyectos o planos de construcción y/o remodelación de espacios, con los costos estimados y proyectados al menos a cinco años.

La estrategia operativa del modelo de pertinencia y factibilidad demanda la participación de los actores involucrados en las tareas relacionadas con el diseño y desarrollo curricular y con la vinculación con el sector social y productivo.

El nivel de participación de las áreas académicas involucradas en la estrategia operativa del Modelo de Pertinencia Académica y factibilidad se describe enseguida:

En la etapa de pertinencia se realizan tres estudios:

a) Estudio de expectativas del nivel medio superior. Las funciones asociadas a este estudio son el diseño metodológico, instrumental, análisis, reportes y difusión de resultados las cuales son desarrolladas por la Secretaría Académica. La aplicación de los instrumentos (formato impreso) queda bajo la responsabilidad de las Facultades y Unidades Académicas.

b) Estudio de egresados y Estudio de Empleadores. Las funciones relacionadas con los dos estudios son el diseño metodológico, instrumental, análisis, reportes y difusión de resultados, las cuales se desarrollan conjuntamente entre la Secretaría Académica y la Secretaría de Vinculación. La aplicación de los instrumentos (en línea) queda bajo la responsabilidad de la Secretaría de vinculación.

c) Estudio de Expertos. La Secretaría Académica es la responsable del Diseño metodológico, instrumental, análisis, reportes y difusión de resultados. La aplicación es responsabilidad de las Facultades y Unidades Académicas.

La etapa de factibilidad requiere de la realización de tres estudios:

a) Estudios retrospectivos y prospectivos de la población escolar de nivel medio superior.

b) Análisis de la capacidad académica de cada programa educativo.

c) Análisis de equipamiento e infraestructura. 
Los tres estudios requieren de la definición de una guía metodológica que establezca los lineamientos para efectuar estudios prospectivos que proyecten el crecimiento gradual de las demandas educativas, así como los requerimientos de la capacidad académica y de infraestructura y equipo; lo cual es responsabilidad de la Secretaría Académica. Con la guía metodológica y la capacitación al personal de las Facultades y Unidades Académicas, éstas se responsabilizan de realizar los estudios correspondientes a la etapa de factibilidad.

Alineado a esta estrategia se están diseñando procedimientos de calidad en los que se detallan los elementos metodológicos, instrumentos y nivel de responsabilidad de la operatividad del modelo de pertinencia académica y factibilidad. Asimismo se están elaborando lineamientos metodológicos que normen el proceso de pertinencia y factibilidad académica en la Universidad.

\section{MATERIAL Y MÉTODOS}

Primeramente se describen los elementos metodológicos de tres de los estudios realizados al momento como son los estudios sobre las preferencias profesionales de estudiantes de educación media superior, el de egresados y empleadores. Los tres estudios tienen un diseño de investigación de corte cuantitativo, descriptivo, transversal y no experimental. (Palella S. \& Martins, 2006) y (Hernández Sampieri, Fernández Collado, \& Baptista Lucio, 2010)

En el caso de la investigación sobre expectativas de formación profesional realizado con estudiantes de nivel medio superior, se seleccionaron a aquellos que cursaban su último periodo escolar en mayo de 2019, tanto de escuelas públicas como privadas, de los diferentes subsistemas de la entidad federativa de Tamaulipas. El universo estuvo conformado por 36,828 estudiantes y se determinó el tamaño muestral utilizando la fórmula para poblaciones menores de 10,000 de (Hernández Sampieri, Fernández Collado, \& Baptista Lucio, 2010), aplicando un nivel de confianza del 95\%, un error estándar del 5\%, una probabilidad a favor de $30 \%$ y una probabilidad de fracaso de $70 \%$. También se optó por un muestreo estratificado considerando la agrupación de los municipios de Tamaulipas según la Región del Estado. Se definió una muestra para cada una de las regiones del Estado quedando un total de 1615 estudiantes distribuidos en 6 zonas: Región Fronteriza (317), Región Valle de San Fernando (195), Región Centro (306), Región Sur (313), Región Mante (279) y Región Altiplano (205).

En el estudio de egresados se tomó como universo el total de alumnos registrados en la Cédula de Egreso en el periodo 2019-2. Se aplicó el instrumento a 840 egresados de la Universidad, sin haber aplicado un marco muestral. La aplicación fue en línea. Por lo que respecta al estudio de opinión de empleadores, se utilizó el instrumento Estudio de las expectativas de los Empleadores, mismo que fue aplicado a un total de 101 empleadores de los egresados de la Universidad de los estados de Tamaulipas, Nuevo León y Guadalajara. Este instrumento también se aplicó en línea, sin un muestreo metodológicamente riguroso.

\section{RESULTADOS}

\section{Del estudio de expectativas de formación profesional de estudiantes del nivel medio superior.}

El estudio fue aplicado a un total de 4,163 estudiantes que cursaban el último periodo, en 74 instituciones públicas y privadas de nivel medio superior, de marzo a mayo de 2019. La presentación de los resultados se hace por las variables de análisis y los ítems que las integraron.

- Análisis de los datos generales.
Edad: Los resultados evidencian que predominan alumnos de 17 y 18 años de edad, lo que equivale al $55,3 \%$ y $36,9 \%$ respectivamente de la muestra.

Sexo y estado civil: es ligera la diferencia en el género. Hay un $54,1 \%$ de sexo femenino y un $45,4 \%$, el resto de la muestra encuestada no indicó su sexo; y, con relación al Estado Civil, un $94 \%$ de los encuestados son solteros.

Situación laboral: De acuerdo a la respuesta de los alumnos encuestados el $77,7 \%$ cuentan con trabajo. 


\section{- Datos socioeconómicos.}

El $78,5 \%$ de la muestra viven en una localidad de tipo urbana. Los principales solventadores financieros son los padres de los estudiantes de media superior, el $48,1 \%$ son padre y madre juntos, el 29,8\% únicamente el padre, y el $16 \%$ únicamente la madre.

\section{- Expectativas profesionales.}

El $96 \%$ de los encuestados tiene interés en continuar sus estudios superiores: $88,1 \%$ en licenciatura, 3,2\% en técnico superior, $4,7 \%$ una carrera técnica; el $4 \%$ no respondieron; y, los que desean estudiar eligieron preferentemente en la modalidad presencial $(91,6 \%)$.

Del total de encuestados, el 53,6\% están interesados en las carreras de la UAT. A este grupo de alumnos se les pidió elegir la carrera de mayor interés. En orden de prioridad se enlistan las 10 carreras de mayor interés: Lic. en Enfermería, Ing. Industrial, Médico Cirujano, Contador Público, Ing. en Sistemas Computacionales, Ing. Agrónomo, Lic. en Psicología, Lic. Negocios Internacionales y Lic. en Derecho. Cabe señalar que algunas las carreras son ofertadas en diferentes campus de nuestra Universidad.

Por otro lado, de los 1,529 estudiantes que opinaron no estar interesados en las carreras que se ofertan en la UAT, su preferencia se centra en las siguientes carreras: Mecatrónica, Ing. Industrial, Médico Cirujano, Contador Público, Psicología, Derecho, Administración de Empresas, Ing. en Sistemas Computacionales, Criminología y Gestión Empresarial.

De este conjunto de estudiantes, se les pidió indicaran la institución de su preferencia y la mayoría de este grupo (115, un 6,3\%), respondieron que estudiarán en la UAT. Lo que evidencia que la carrera que buscan no se ofrece en la institución, pero que sí continuarían sus estudios en la UAT si estuviese su carrera. Las Instituciones de competencia para la UAT son: Universidad Autónoma De Nuevo León (UANL), Instituto De Ciencias y Estudios Superiores De Tamaulipas (ICEST), Instituto Tecnológico de Matamoros, Instituto Tecnológico de Reynosa, Universidad Tecnológica de Matamoros (UTM), Universidad Tecnológica de Tamaulipas (UTT), Universidad Tecnológica (UT), Instituto Tecnológico de Cd. Victoria, Universidad México Americana del Norte (UMAN), Instituto Tecnológico de Nuevo Laredo, Universidad del
Valle De México (UVM).

Resultó significativo el hecho de que ante la posibilidad de no existir la carrera de interés en su localidad, un $69 \%$ de los estudiantes optarían por buscar una opción similar en la misma ciudad.

\section{Del estudio de egresados y empleadores.}

La información obtenida de los empleadores evidencia su percepción respecto al desempeño de egresados de la UAT. Un $74,3 \%$ de los empleadores encuestados consideran que el desempeño de egresados de la Universidad es bueno y muy bueno. Sin embargo su opinión con respecto a egresados de otras IES no es tan diferente dado que un $69,3 \%$ de empleadores opina que el desempeño de estos egresados fue buena y muy buena.

Por otro lado, en el caso de la opinión de los egresados de la Universidad se les preguntó acerca de la satisfacción que tienen respecto a la formación recibida en la licenciatura; en este caso se observó que el $68,4 \%$ se encuentra satisfecho y muy satisfecho con la formación recibida por la UAT. Sin embargo, para futuras investigaciones es necesario indagar la causa por la que el $23,1 \%$ de la muestra está regularmente satisfecha con la formación brindada.

Con los resultados obtenidos de ambos instrumentos, se observa coincidencia en cuanto a la opinión de egresados y empleadores con respecto a las Competencias, Conocimientos y Habilidades Técnicas que se requieren en el campo laboral. Se enlistan enseguida aquellas que fueron seleccionadas por ambos.

\section{- Sobre las Competencias (Habilidades Blandas)}

Sobre este tipo de competencias se encontró coincidencia de importancia y utilidad en el trabajo lo siguiente: Comunicación, Orientación al servicio, Solución de problemas complejos, Flexibilidad cognitiva (aprendizaje continuo), Inteligencia emocional, Actitud emprendedora y Liderazgo (administración de personal). Este grupo de competencias se consideraron indispensables para profesionistas de cualquier campo disciplinar.

\section{- Sobre conocimientos y habilidades técnicas}

Aquí se encontró coincidencia en competencias que, desde la perspectiva de egresados y 
empleadores, son de carácter más técnico, pero que definitivamente también pueden ser desarrolladas por profesionistas de cualquier campo disciplinar y son: Administración de empresas, Inglés u otro idioma, Mercadotecnia y ventas, Análisis financiero, Análisis estadístico, Habilidades informáticos (tics, programación, big data), Habilidades de investigación, Habilidades de ingeniería, Leyes y gobierno, Medicina y psicología. Lo anterior denota la necesidad de formar profesionistas cada vez más generalistas con competencias útiles en cualquier campo profesional y que le permitan ir marcando trayectorias laborales y profesionales transdiciplinares.

\section{CONCLUSIONES}

La demanda de formación de profesionistas pertinentes y de calidad ha sido un desafío planteado desde el ámbito internacional y nacional, lo anterior derivado de estudios realizados por la OECD (OCDE, 2019), (SalazarXirinachs, s/f), (Pérez, S/f), (Carmona Hinojosa, 2017), (Ortega Santos, 2017) así como de organismos que establecen políticas educativas de educación superior en México como la ANUIES (ANUIES, 2018).

La educación superior se enfrenta ante la necesidad de promover el desarrollo de habilidades blandas para lograr un mejor enfoque laboral y que los egresados estén mejor preparados para cumplir las tareas específicas de trabajo en su especialidad; el desarrollo de competencias profesionales 4.0, para impulsar la industria 4.0; fortalecer la formación de habilidades intelectuales y competencias de carácter transversal; la formación de recursos humanos altamente calificados y versátiles con capacidad para adaptarse con facilidad y rapidez a las nuevas circunstancias del mundo laboral.

Con base en lo anterior, la Universidad Autónoma de Tamaulipas diseñó el modelo integral de pertinencia y factibilidad académica a fin de identificar oportunamente los nuevos requerimientos profesionales, y con ello, facilitar los procesos de actualización curricular que aseguren una oferta educativa pertinente y con ello el aseguramiento de la calidad de sus servicios.

Los avances logrados al momento es un proceso de trabajo conjunto con lineamientos metodológicos e instrumentales establecidos desde la administración central de la Universidad y una mayor sistematización de la información y sus resultados. Los resultados de los estudios realizados al momento han permitido:

Con relación al estudio de las expectativas profesionales de estudiantes del nivel medio superior, identificar aquellas carreras que la Universidad ofrece y que son de interés para los estudiantes; pero también se identificaron nuevas opciones profesionales que permitirán a la Universidad redefinir su oferta educativa en los diferentes municipios del estado en donde tiene presencia.

Los resultados preliminares de los estudios de egresados y empleadores han sido relevantes en la medida en que actualmente la Universidad se encuentra en un proceso de actualización de los programas educativos, y, en este sentido, la opinión de estos informantes clave permite ir identificando competencias transversales y profesionales que son indispensables en el desempeño laboral. Aspecto que ha ido retroalimentando el trabajo de los equipos de diseño curricular de las distintas Facultades. De ahí la necesidad de repensar el nuevo modelo educativo de la Universidad basado en un diseño curricular más flexible, que considere las certificaciones de competencias profesionales, la posibilidad de reconocer créditos de estudiantes que estén próximos a abandonar una licenciatura a través de una salida lateral, la transitabilidad de estudiantes entre programas educativos de la misma institución favoreciendo la interdisciplinariedad y la integración de enfoques formativos integradores e innovadores de enseñanza.

Finalmente, y derivado del modelo de pertinencia y factibilidad, la Universidad se encuentra trabajando en la realización de cinco foros de expertos, a realizarse en cinco de las ciudades del estado con mayor dinamismo y desarrollo económico, los cuales tendrán la finalidad de conocer la opinión que éstos expertos, conocedores de los polos de desarrollo del estado de Tamaulipas, tienen acerca de las demandas de formación de nuevos profesionistas y especialistas que puedan atender los retos de desarrollo del estado.

Con lo anterior, se concluye que si bien el modelo y su aplicación es aún incipiente en la UAT, ha empezado a tener resultados preliminares muy interesantes dado que la toma de decisiones va más allá de un interés político, sustentándose ahora en lineamientos y directrices emanadas de 
planes de desarrollo internacional, nacional, estatal e institucional, demandando con ello un cambio de cultura académica y organizacional en los distintos niveles de la institución, desde las dependencias de la Universidad, en las
Facultades, profesores y administrativos y atender con ello tres de los grandes retos planteados a la educación superior: mayor pertinencia, calidad y cobertura, pero con responsabilidad social.

\section{REFERENCIAS BIBLIOGRÁFICAS}

ANUIES. (2018). Visión y acción 2030 Propuesta de la ANUIES para renovar la educación superior en México Diseño y concertación de políticas públicas para impulsar el cambio institucional. México: ANUIES.

Carmona Hinojosa, L. (2017). ¿Estamos preparando a nuestros jóvenes para el futuro laboral que les espera? Revista de esudios de juventud no. 17.

Hernández Sampieri, R., Fernández Collado, C., \& Baptista Lucio, P. (2010). Metodología de la investigación. México: Mc GrawHill/Interamericana Editores S. A de C.V.

Lladó Lárraga, D. M., Navarro Leal, M., \& Sánchez Rodríguez, L. I. (2013). Competencias profesionales y empleabilidad en el contexto de la flexibilidad laboral. Estados Unidos: Palibrio.

OCDE. (01 de 01 de 2019). Higher education in Mexico:Labour market relevance and outcomes, higher education. París, Francia.

Organización de las Naciones Unidas para la Educación, la Ciencia y la Cultura. (2015). UNESCO. Obtenido de UNESCO: www.unesco.org
Ortega Santos, C. (2017). Desarrollo de habilidades blandas desde edades tempranas. Guayaquil, Ecuador: Centro de Publicaciones-Universidad ECOTEC.

Palella S., S., \& Martins, F. (2006). Metodología de la investigación cuantitativa. Caracas, Venezuela: FEDUPEL.

Pérez, M. d. (S/f). Davos y la cuarta revolución Industrial. https://reunir.unir.net > bitstream > handle > Davos y la cuarta revolucion i...

Salazar-Xirinachs, J. (s/f). El futuro del trabajo, el empleo y las competencias en América Latina y el Caribe. Revista Pensamiento Iberoamericano. https://www.segib.org $>$ wp-content > uploads > 07-JMS-X.

Universidad Autónoma de Tamaulipas. (14 de mayo de 2018). Universidad Autónoma de Tamaulipas. (U. A. Tamauipas, Ed.) Recuperado el 14 de Noviembre de 2019, de http://www.uat.edu.mx

Recibido: 25 mayo 2019| Aceptado: 26 julio 2019 